\title{
GERMINABILIDAD Y DESARROLLO INICIAL DE NUEVOS HÍBRIDOS INTRAESPECÍFICOS DE Theobroma cacao $\mathrm{L}$.
}

\section{GERMINABILITY AND INITIAL DEVELOPMENT OF NEW INTRA-SPECIFIC HYBRIDS OF Theobroma cacao L.}

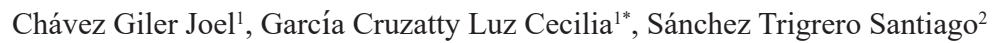 \\ ${ }^{1}$ Facultad de Ingeniería Agronómica, Universidad Técnica de Manabí, Manabí-Ecuador, 130105 \\ ${ }^{2}$ Investigador Independiente \\ *Autor de correspondencia:luz.garcia@utm.edu.ec
}

Rec.: 07.11.2019. Acept.: 02.10.2020.

Publicado el 30 de diciembre de 2020

\section{Resumen}

$\mathrm{E}^{1}$ objetivo de esta investigación fue evaluar la germinabilidad de semillas y el desarrollo inicial de híbridos interclonales de Theobroma cacao L. El ensayo se estableció en el campus experimental "La Teodomira", Manabí, Ecuador. Se realizaron cruzamientos intraespecíficos entre cinco genotipos de T. cacao, los frutos obtenidos se cosecharon aproximadamente a los 180 días. Las variables estudiadas fueron: porcentaje de germinación y de emergencia, altura de planta, diámetro de tallo, número de hojas y contenido de clorofila en el área foliar. Se obtuvo un $97.8 \%$ de germinación a los ocho días luego de desmulización e imbibición y un $86.5 \%$ de emergencia, registrándose un mayor porcentaje de germinación y emergencia en la progenie derivada del cruce L11-H19 x EET-103. A los 120 días, el mayor diámetro de tallo se registró en la familia EET-558 x CCN-51 y la mayor altura en la familia EET-103 x L11-H19. En promedio se obtuvo 15 hojas por planta y 43.5 de clorofila SPAD a los 120 días. El contenido de clorofila en las hojas está condicionado por la edad fisiológica de las plántulas; no obstante, para futuras investigaciones se recomienda realizar evaluación en campo de estas progenies, en especial de aspectos fisiológicos relacionados con la producción de hojas y contenido de clorofila, pues estos podrían ser indicadores para selección de genotipos promisorios.

Palabras claves: Cruzamiento, Genotipo, Germinación, Habilidad combinatoria, Semilla.

\begin{abstract}
$T$ he objective of this research was to evaluate the germinability of seeds and the initial development of interclonal hybrids of Theobroma cacao L. The trial was established in the experimental campus "La Teodomira", Manabí, Ecuador. Intraspecific crosses were made between five genotypes of $T$. cacao, the fruits obtained were harvested at approximately 180 days. The variables studied were: germination and emergence percentage, plant height, stem diameter, number of leaves and chlorophyll content in the leaf area. A $97.8 \%$ germination was obtained at eight days after demulization and imbibition and an $86.5 \%$ emergence, registering a higher percentage of germination and emergence in the progeny derived from the L11-H19 x EET-103 cross. At 120 days, the greatest stem diameter was recorded in the EET-558 x CCN-5 family and the greatest height in the EET-103 x L11-H19 family. On average, 15 leaves per plant and 43.5 of SPAD chlorophyll were obtained at 120 days. The chlorophyll content in the leaves is determined by the physiological age of the seedlings. However, for future research it is recommended to carry out field evaluation of these progenies, especially of physiological aspects involved with the production of leaves and chlorophyll content, since these could be indicators for the selection of promising genotypes.
\end{abstract}

Keywords: Crossing, genotype, germination, combinatory hability, seed 


\section{Introducción}

$\mathrm{E}$ 1 cacao (Theobroma cacao L; Malvaceae) es un cultivo perenne de alto valor comercial en el mundo y representa uno de los rubros más importantes para las regiones tropicales de América Latina, África y Asia (Sánchez, 2016). Ecuador es el principal productor de cacao fino de aroma, aportando un 54\% de la producción mundial. Sin embargo, la producción nacional no supera los $300 \mathrm{~kg} \mathrm{ha}^{-1}$ año $^{-1}$ (Acebo, 2016). Los bajos rendimientos en Ecuador se han atribuido generalmente a un gran número de factores, tanto endógenos (genéticos y fisiológicos) como externos (variaciones ambientales y manejo agronómico) (Quiroz \& Amores 2002).

En Ecuador es importante desarrollar nuevas variedades de $T$. cacao con la finalidad de obtener genotipos tolerantes a las principales enfermedades que amenazan el cultivo y altamente productivos, considerando también la conservación de la calidad organoléptica propia del cacao ecuatoriano. La generación de nuevos genotipos de $T$. cacao trae consigo la necesidad de estudiar el comportamiento agronómico de plantas, desde su estado inicial hasta su producción en campo, así mismo, conocer su interacción en diferentes ambientes. Cabe destacar, que una buena selección de plántulas en estado de vivero, facilitará el óptimo desarrollo de las plantas en su sitio definitivo.

El presente estudio tiene como objetivo; evaluar la germinabilidad y el desarrollo inicial de híbridos interclonales de T. cacao. Con base en lo mencionado, es necesario realizar un seguimiento permanente a las progenies derivadas de nuevos cruzamientos, debido a que la identificación de genotipos promisorios marca pautas en la selección de materiales élites.

\section{Materiales y métodos}

$\mathrm{Z}^{1 \text { trabajo fue realizado durante enero del } 2018 \text { hasta }}$ febrero de 2019, en la estación experimental "La Teodomira", Universidad Técnica de Manabí, Ecuador $\left(80^{\circ} 26^{\prime} 22\right.$ " de longitud oeste y $01^{\circ} 04^{\prime} 15^{\prime \prime}$ de latitud sur), la misma que se encuentra a una altura de 60 msnm, con una temperatura media anual de $26.5^{\circ} \mathrm{C}$, precipitación de $669 \mathrm{~mm}$ anuales y radiación de 1,146 horas luz por año.

Los híbridos interclonales fueron obtenidos a partir de cruzamientos entre cinco genotipos de T. cacao tipo Nacional y un Trinitario (L26-H64, EET-103, L11-H19, L21-H38, EET-558 y CCN-51 (Trinitario)). Estos genotipos en estudios previos presentaron altos niveles de producción, sanidad vegetal y buena adaptabilidad en la zona de estudio (Sánchez et al., 2016).

Para la obtención de híbridos interclonales se realizaron cruzamientos asistidos, siguiendo un sistema dialélico incompleto (Cuadro 1). Por cada genotipo se encapsularon 20 botones florales en preantesis, usando tubos Eppendorf ( $2 \mathrm{~mL}$ ). Transcurridas las 24 horas después del encapsulado, cuando las flores se encontraban en antesis, se realizaron las respectivas polinizaciones manuales. Para las polinizaciones se consideró el periodo de receptividad estigmática, comprendido entre las 10:00 a.m a 13:00 p.m (Mena \& García, 2017); 48 horas después de la polinización se retiraron los tubos Eppendorf y se registró el número de flores fecundadas. Los frutos obtenidos a partir de los cruzamientos se cosecharon a los 180 días aproximadamente, cuando se evidenció la madurez fisiológica.

Cuadro 1. Sistema de cruzamiento dialélico incompleto para obtención de híbridos intraespecíficos de Theobroma cacao $\mathrm{L}$

\begin{tabular}{ccccccc}
\hline L26-H64 & L21-H38 & EET-103 & EET-558 & L11-H19 & CCN-51 \\
\hline L21-H38 & $\mathrm{X}$ & & & & $\mathrm{X}$ & \\
EET-103 & & $\mathrm{X}$ & & & $\mathrm{X}$ & $\mathrm{X}$ \\
EET-558 & & & $\mathrm{X}$ & & & \\
L11-H19 & $\mathrm{X}$ & & $\mathrm{X}$ & $\mathrm{X}$ & & $\mathrm{X}$ \\
CCN-51 & $\mathrm{X}$ & & $\mathrm{X}$ & $\mathrm{X}$ & $\mathrm{X}$ & \\
\hline
\end{tabular}

Letras " $\mathrm{X}$ " indican cruzamiento entre genotipos. 
Una vez obtenidos los frutos, se extrajeron las semillas, se les retiró el mucílago y el epispermo, para posteriormente colocarlas en imbibición $(1,000 \mathrm{~mL}$ de agua). El porcentaje de germinación fue evaluado cada 24 horas, hasta que no se observó germinación por 48 horas seguidas. Se consideró como germinadas a las semillas que mostraban la emisión radícular.

Se escogieron al azar 10 individuos por familia, en los cuales se midió las siguientes variables: altura de planta $(\mathrm{cm})$, diámetro del tallo $(\mathrm{cm})$, número de hojas y contenido de clorofila en las hojas a los 30, 60, 90 y 120 días. La altura se midió desde la superficie del sustrato hasta el ápice terminal de la planta, usando una cinta métrica. El diámetro del tallo se tomó cinco centímetros arriba de la superficie del sustrato, sobre la cicatriz cotiledonal, para esto se utilizó un calibrador digital. Cada 30 días se contabilizó el número de hojas nuevas, las mismas que debían estar totalmente desplegadas. El contenido de clorofila de hojas se obtuvo usando un medidor de pigmentación SPAD-502, en cuatro hojas por planta, ubicadas en estrato medio de la misma. Estas medidas se obtuvieron del haz, del lado izquierdo y derecho, entre la nervadura central de la hoja.

\section{Análisis estadístico}

El ensayo se estableció siguiendo un diseño completamente al azar. Para todas las variables medidas, se realizó un análisis de varianza y contraste de medias utilizando la prueba de Tukey $(p \leq 0.05)$, previamente se corroboró que los datos cumplían con los supuestos de normalidad, homocedasticidad e independencia de variables. Se realizó una regresión lineal y se calculó el índice de correlación de Pearson entre el número de hojas y las variables diámetro del tallo y altura de la planta. De igual forma, se calculó el índice de correlación entre el contenido de clorofila y la edad de las plantas. Para estos análisis se utilizó el software Statistica 7.0.

\section{Resultados y discusión}

$\mathrm{U}$ n $90.1 \%$ de las semillas germinaron, siendo la familia derivada del cruce L11-H19 x EET-103 la de mayor germinación, 97.8\% (Cuadro 2). Estos resultados son superiores a los obtenidos por López \& Gil (2017), quienes obtuvieron $88.9 \%$ de germinación, diferencia que se puede atribuir al origen de las semillas y al tratamiento pregerminativo de desmulización. Adu et al (2017), mencionan que la desmulización de las semillas en $T$. cacao incrementa la germinación entre un 80 y $100 \%$ comparado con las semillas de mucilago no removido.

El promedio de emergencia fue $86.5 \%$, siendo mayor en la familia procedente del cruce L11-H19 x EET-103 (95.7\%) y el menor para los individuos provenientes del cruzamiento L11-H19 x CCN-51 (62.2\%) (Cuadro 2). Estos resultados son superiores a los obtenidos por López \& Gil (2017), quienes obtuvieron un 71.1\% de emergencia en semilla con desmulización, diferencias que podrían relacionarse con el sustrato y el origen de las semillas. El sustrato adecuadamente proporcionado brinda las condiciones físicas y nutricionales para una mayor emergencia, sin embargo, el vigor de las semillas depende también de las caracteristicas heredadas por sus parentales. Aunque la porosidad del sustrato afecta la capacidad del intercambio gaseoso del medio, disminuyendo el oxígeno que necesita la semilla para emerger del suelo (Ortega et al., 2010).

Cuadro 2. Germinación y emergencia de semillas en 10 familias de híbridos intraespecíficos de Theobroma cacao L., en la finca experimental "La Teodomira" Manabí

\begin{tabular}{lcc}
\hline \multicolumn{1}{c}{ Familias } & Germinación (\%) & Emergencia (\%) \\
\hline L11-H19 x EET-103 & 97.8 & 95.7 \\
EET-558 x EET-103 & 92.4 & 87.7 \\
EET-558 x L11-H19 & 83.1 & 67.2 \\
EET-103 x L11-H19 & 90.0 & 80.0 \\
L11-H19 x CCN-51 & 78.3 & 62.2 \\
L11-H19 x EET-558 & 96.4 & 92.5 \\
EET-103 x CCN-51 & 89.2 & 81.9 \\
EET-558 x CCN-51 & 91.6 & 86.0 \\
EET-103 x EET-558 & 88.4 & 80.7 \\
CCN-51 x EET-103 & 94.6 & 94.6 \\
\hline $\bar{X} \pm$ Sd & $90.1( \pm 5.9)$ & $86.5( \pm 2.8)$ \\
\hline
\end{tabular}


Las plántulas evaluadas a los 30 días presentaron un diámetro promedio de $1.69 \mathrm{~mm}$, siendo la familia derivada del cruce CCN-51 x EET-103 la que presentó mayor diámetro (1.91 $\pm 0.2 \mathrm{~mm}$ ) (Cuadro 3). Sin embargo, a los 120 días el promedio fue de $4.29 \mathrm{~mm}$, siendo la familia procedente de cruce EET-558 x CCN51 la que presentó mayor crecimiento $(4.69 \pm 0.5 \mathrm{~mm}$ ) (Cuadro 3). Los resultados obtenidos para esta variable fueron inferiores a los reportados por Sarmiento et al (2011), quienes obtuvieron un diámetro de $5.14 \mathrm{~mm}$ a los 93 días, en semillas del clon IMC-67 en la Amazonía colombiana. De igual forma, se evidenciaron mayores resultados en evaluaciones del crecimiento inicial del clon EET-116 en el Litoral ecuatoriano (Llerena et al., 2017). Según Ortega et al (2010), utilizar un sustrato de buena fuente mineral favorece el desarrollo del diámetro; no obstante, esta característica podría depender de la ganacia genética que los individuos hayan adquirido mediante la heredabilidad de sus parentales.

La variable altura de planta difiere entre las familias evaluadas, en promedio fue de $18.8 \mathrm{~cm}$ a los 30 días y de $39.2 \mathrm{~cm}$ a los 120 días (Cuadro 4). Los individuos de la familia EET-103 x L11-H19 presentaron el mejor crecimiento en altura $(47.80 \pm 4.9)$ y el menor crecimiento fue registrado para la familia EET-558 x EET-103 (34.64 \pm 4.6 ). Los resultados obtenidos son superiores a los reportados por Sarmiento et al (2011), quienes evaluaron plantas provenientes del clon IMC67 ( $15.1 \mathrm{~cm}$ a los 23 días y $24.9 \mathrm{~cm}$ a los 93 días). Sin embargo, son similares a lo reportado por Llerena et al (2017) a los 60 días, en plantas descendientes del clon
EET-116 (26.4 cm). Cuéllar y Herrera (2010) sostienen que utilizar cubierta promueve la elongación del tallo, lo que genera plantas muy suculentas y poco lignificadas, pero en este estudio todas las familias fueron evaluadas en la misma condición, por lo que las diferencias podrían atribuirse a la variabilidad intra e interfamiliar.

En promedio se obtuvieron cuatro hojas a los 30 días, y 13 hojas a los 120 días. La familia procedente del cruzamiento EET-103 x L11-H19 tuvo el mayor número de hojas durante el periodo de evaluación (Cuadro 5). Los resultados obtenidos son similares a los reportados por Sarmiento et al (2011), pues obtuvieron 4.6 hojas a los 23 días y 10 hojas a los 65 días, en genotipos descendientes del clon IMC-67. Por su lado, Almeida (2012) afirma que la sombra utilizada puede aportar en el desarrollo de las hojas, pues reducir la luminosidad aumenta el área foliar, mostrando una mayor inversión en la producción de vástago y hojas. Lo cual no se pudo corroborar en este estudio, debido a que todos los individuos se evaluaron bajo la misma condición de sombra.

La mejor HCG se presentó en el clon EET-103 como madre con valores de $4.55 \mathrm{~mm}$ en el diámetro, $39.5 \mathrm{~cm}$ de altura y 14 hojas promedio. Sin embargo, se observa superioridad de EET-558 como padre con promedios de $4.4 \mathrm{~mm}$ de diámetro, $36.6 \mathrm{~cm}$ de altura de planta y 14 en el número de hojas (Cuadro 6). Según Camacho et al (2006), los genotipos que tienen altas concentraciones de genes deseados son los más indicados para formar nuevas poblaciones mejoradas, debido a que tienen mayor capacidad de trasmitir estos genes a sus progenies.

Cuadro 3. Habilidad combinatoria específica en el diámetro de tallo $(\mathrm{mm})$ en 10 familias de híbridos intraespecíficos de Theobroma cacao L., a los 30, 60, 90 y 120 días

\begin{tabular}{lcccc}
\hline \multirow{2}{*}{ Familias } & \multicolumn{4}{c}{ Diámetro de tallo $(\mathrm{mm})$} \\
\cline { 2 - 5 } & 30 días & 60 días & 90 días & 120 días \\
\hline L11-H19 x EET-103 & $1.60( \pm 0.1) \mathrm{b}$ & $2.78( \pm 0.3) \mathrm{a}$ & $3.20( \pm 0.6) \mathrm{c}$ & $3.87( \pm 0.6) \mathrm{b}$ \\
EET-558 x EET-103 & $1.60( \pm 0.1) \mathrm{b}$ & $2.63( \pm 0.3) \mathrm{a}$ & $3.36( \pm 0.5) \mathrm{c}$ & $3.88( \pm 0.7) \mathrm{b}$ \\
EET-558 x L11-H19 & $1.62( \pm 0.3) \mathrm{b}$ & $2.22( \pm 0.2) \mathrm{b}$ & $3.28( \pm 0.7) \mathrm{c}$ & $3.67( \pm 0.9) \mathrm{b}$ \\
EET-103 x L11-H19 & $1.60( \pm 0.1) \mathrm{b}$ & $2.88( \pm 0.3) \mathrm{a}$ & $4.24( \pm 0.4) \mathrm{a}$ & $4.66( \pm 0.4) \mathrm{b}$ \\
L11-H19 x CCN-51 & $1.60( \pm 0.1) \mathrm{b}$ & $2.33( \pm 0.6) \mathrm{b}$ & $3.70( \pm 0.6) \mathrm{c}$ & $4.48( \pm 0.8) \mathrm{a}$ \\
L11-H19 x EET-558 & $1.59( \pm 0.2) \mathrm{b}$ & $2.48( \pm 0.6) \mathrm{b}$ & $3.83( \pm 0.8) \mathrm{b}$ & $4.53( \pm 0.7) \mathrm{a}$ \\
EET-103 x CCN-51 & $1.88( \pm 0.2) \mathrm{a}$ & $2.54( \pm 0.3) \mathrm{b}$ & $3.58( \pm 0.4) \mathrm{c}$ & $4.56( \pm 0.9) \mathrm{a}$ \\
EET-558 x CCN-51 & $1.76( \pm 0.3) \mathrm{a}$ & $2.55( \pm 0.3) \mathrm{b}$ & $4.14( \pm 0.3) \mathrm{a}$ & $4.69( \pm 0.5) \mathrm{a}$ \\
EET-103 x EET-558 & $1.66( \pm 0.1) \mathrm{b}$ & $2.37( \pm 0.5) \mathrm{b}$ & $3.81( \pm 0.9) \mathrm{b}$ & $4.44( \pm 0.8) \mathrm{a}$ \\
CCN-51 x EET-103 & $1.91( \pm 0.2) \mathrm{a}$ & $2.73( \pm 0.3) \mathrm{a}$ & $3.75( \pm 0.6) \mathrm{b}$ & $4.12( \pm 0.6) \mathrm{a}$ \\
\hline$\overline{\mathrm{X}} \pm$ Sd & $1.68( \pm 0.2)$ & $2.56( \pm 0.4)$ & $3.74( \pm 0.7)$ & $4.29( \pm 0.7)$ \\
\hline
\end{tabular}

Letras distintas indican diferencia estadística, Tukey $(\mathrm{p} \leq 0.05)$. 
Cuadro 4. Habilidad combinatoria específica en altura de planta en $\mathbf{1 0}$ familias de híbridos intraespecíficos de Theobroma cacao L., a los 30, 60, 90 y 120 días

\begin{tabular}{lcccc}
\hline \multirow{2}{*}{ Familias } & \multicolumn{4}{c}{ Altura de planta $(\mathrm{cm})$} \\
\cline { 2 - 5 } & 30 días & 60 días & 90 días & 120 días \\
\hline L11-H19 x EET-103 & $19.4( \pm 1.5) \mathrm{a}$ & $22.6( \pm 2.5) \mathrm{c}$ & $32.77( \pm 3.4) \mathrm{b}$ & $38.82( \pm 6.4) \mathrm{c}$ \\
EET-558 x EET-103 & $19.9( \pm 1.4) \mathrm{a}$ & $23.4( \pm 2.4) \mathrm{b}$ & $31.45( \pm 3.9) \mathrm{c}$ & $34.64( \pm 4.6) \mathrm{e}$ \\
EET-558 x L11-H19 & $18.9( \pm 1.5) \mathrm{b}$ & $22.0( \pm 2.0) \mathrm{c}$ & $28.51( \pm 2.5) \mathrm{d}$ & $37.97( \pm 2.3) \mathrm{c}$ \\
EET-103 x L11-H19 & $20.0( \pm 2.5) \mathrm{a}$ & $25.1( \pm 4.4) \mathrm{a}$ & $36.15( \pm 5.7) \mathrm{a}$ & $47.80( \pm 4.9) \mathrm{a}$ \\
L11-H19 x CCN-51 & $18.9( \pm 2.2) \mathrm{b}$ & $21.5( \pm 4.0) \mathrm{c}$ & $28.40( \pm 4.1) \mathrm{d}$ & $38.04( \pm 6.4) \mathrm{c}$ \\
L11-H19 x EET-558 & $18.1( \pm 1.2) \mathrm{b}$ & $21.5( \pm 2.5) \mathrm{c}$ & $29.19( \pm 5.1) \mathrm{c}$ & $36.48( \pm 5.6) \mathrm{d}$ \\
EET-103 x CCN-51 & $18.2( \pm 2.0) \mathrm{b}$ & $22.2( \pm 6.2) \mathrm{b}$ & $30.47( \pm 5.7) \mathrm{c}$ & $42.18( \pm 6.4) \mathrm{b}$ \\
EET-558 x CCN-51 & $18.5( \pm 1.4) \mathrm{b}$ & $24.5( \pm 3.4) \mathrm{a}$ & $33.74( \pm 4.8) \mathrm{b}$ & $41.40( \pm 5.6) \mathrm{b}$ \\
EET-103 x EET-558 & $17.3( \pm 1.6) \mathrm{c}$ & $21.1( \pm 2.7) \mathrm{c}$ & $30.18( \pm 4.5) \mathrm{c}$ & $36.90( \pm 3.9) \mathrm{d}$ \\
$\mathrm{CCN}-51 \times$ EET-103 & $19.5( \pm 2.4) \mathrm{a}$ & $20.0( \pm 2.2) \mathrm{c}$ & $30.54( \pm 5.2) \mathrm{c}$ & $38.71( \pm 3.9) \mathrm{c}$ \\
\hline$\overline{\mathrm{X}} \pm$ Sd & $18.8( \pm 0.8)$ & $22.4( \pm 1.5)$ & $31( \pm 2.6)$ & $39.2( \pm 1.3)$ \\
\hline
\end{tabular}

Letras distintas indican diferencia estadística, Tukey $(\mathrm{p} \leq 0.05)$.

Cuadro 5. Habilidad combinatoria específica en el número de hojas de híbridos interclonales de Theobroma cacao L., a los 30, 60, 90 y 120 días después de la siembra. Los promedios corresponden a la media de 10 individuos por familia

\begin{tabular}{lcccc}
\hline \multicolumn{1}{c}{ Familias } & \multicolumn{4}{c}{ Número de hojas } \\
\cline { 2 - 5 } & 40 & 60 & 90 & 120 \\
\hline L11- H19 x EET-103 & $4.6( \pm 0.8) \mathrm{a}$ & $6.5( \pm 1.2) \mathrm{c}$ & $9.6( \pm 1.8) \mathrm{d}$ & $12.2( \pm 3.2) \mathrm{c}$ \\
EET-558 x EET-103 & $4.6( \pm 0.8) \mathrm{a}$ & $6.9( \pm 1.1) \mathrm{c}$ & $8.4( \pm 1.4) \mathrm{d}$ & $11.2( \pm 1.7) \mathrm{d}$ \\
EET-558 x L11-H19 & $4.2( \pm 1.1) \mathrm{b}$ & $6.6( \pm 1.3) \mathrm{c}$ & $9.2( \pm 2.0) \mathrm{d}$ & $11.9( \pm 1.5) \mathrm{d}$ \\
EET-103 x L11-H19 & $4.6( \pm 0.8) \mathrm{a}$ & $8.0( \pm 1.5) \mathrm{a}$ & $12.1( \pm 1.6) \mathrm{a}$ & $15.3( \pm 2.2) \mathrm{a}$ \\
L11-H19 x CCN-51 & $4.0( \pm 0.6) \mathrm{b}$ & $6.8( \pm 1.3) \mathrm{c}$ & $10.1( \pm 2.1) \mathrm{c}$ & $12.4( \pm 2.8) \mathrm{c}$ \\
L11-H19 x EET-558 & $4.3( \pm 1.0) \mathrm{b}$ & $7.0( \pm 2.0) \mathrm{b}$ & $12.0( \pm 1.8) \mathrm{a}$ & $13.2( \pm 2.7) \mathrm{b}$ \\
EET-103 x CCN-51 & $4.5( \pm 0.7) \mathrm{a}$ & $7.5( \pm 1.2) \mathrm{b}$ & $11.0( \pm 1.5) \mathrm{b}$ & $13.1( \pm 2.3) \mathrm{b}$ \\
EET-558 x CCN-51 & $4.3( \pm 0.4) \mathrm{b}$ & $7.6( \pm 1.1) \mathrm{b}$ & $11.6( \pm 1.2) \mathrm{b}$ & $13.3( \pm 2.4) \mathrm{b}$ \\
EET-103 x EET-558 & $4.4( \pm 0.8) \mathrm{a}$ & $7.2( \pm 1.3) \mathrm{b}$ & $11.8( \pm 2.0) \mathrm{b}$ & $15.2( \pm 2.0) \mathrm{a}$ \\
CCN-51 x EET-103 & $4.2( \pm 1.3) \mathrm{b}$ & $6.7( \pm 1.2) \mathrm{c}$ & $10.6( \pm 1.1) \mathrm{c}$ & $12.0( \pm 1.5) \mathrm{c}$ \\
\hline$\overline{\mathrm{X}} \mathrm{Sd}$ & $4.4( \pm 0.9)$ & $7.8( \pm 1.4)$ & $10.6( \pm 2.0)$ & $13( \pm 2.61)$ \\
\hline
\end{tabular}

Letras distintas indican diferencia estadística, Tukey $(\mathrm{p} \leq 0.05)$.

Cuadro 6. Habilidad combinatoria general (HCG) a los 120 días en el diámetro de tallo, altura de planta y número de hojas en híbridos interclonales de Theobroma cacao $\mathrm{L}$.

\begin{tabular}{ccccccc}
\hline \multicolumn{7}{c}{$q$} \\
Familia & Diámetro (mm) & Altura (cm) & $\begin{array}{c}\text { Número } \\
\text { hojas }\end{array}$ & Diámetro (mm) & Altura (cm) & $\begin{array}{c}\text { Número } \\
\text { hojas }\end{array}$ \\
\hline EET-558 & 4.08 & 38.0 & 12.1 & 4.4 & 36.6 & 14.3 \\
L11-H19 & 4.29 & 37.7 & 12.6 & 4.1 & 42.9 & 13.6 \\
EET-103 & 4.55 & 39.5 & 14.6 & 3.9 & 37.4 & 11.8 \\
CCN-51 & 4.12 & 38.7 & 12.0 & 4.5 & 40.5 & 12.9 \\
\hline
\end{tabular}


Hay una fuerte correlación entre la variables número de hojas y altura del tallo $(r=0.67)$. El número de hojas y el diámetro presenta una correlación moderada $(r=$ 0.37) (Figura 1). Según Contreras y Velázquez (1999) la fuerte corelación entre la altura y el número de hojas en condiciones de vivero se ve favorecida por el uso de sombra, pues las plantas tienden a aumentar su crecimiento aéreo cuando se encuentra a intensidades bajas de luz.

El promedio de clorofila incrementó en el tiempo, a los 30 días fue 34.8 SPAD y de $43.5 \pm 1.2$ a los 120 días. Los individuos de la familia EET-558 x EET-103 tuvieron mayor cantidad de clorofila durante el periodo de estudio, con valores desde $36.8 \pm 2.7$ a los 30 días hasta $43.7 \pm 0.2$ a los 120 días SPAD (Cuadro 7). Los resultados son inferiores a los obtenidos por Motomiya et al (2009), en una especie perteneciente a la misma familia (Gossypium hirsutum L; Malvaceae) en Brasil, 49.53 SPAD a los 85 días en condiciones de campo.
El incremento de la clorofila está vinculado con la disponibilidad de nitrógeno y el uso de sombra, según Carvalho et al (2006), las plantas expuestas a baja radiación presenta un mejor desarrollo de granas, lo que aumenta relativamente el contenido de clorofila.

Hay una relación directa positiva entre el contenido de clorofila en las hojas y la edad de las plántulas $\left(r^{2}\right.$ 0.94) (Figura 2). Esto podría ser atribuido a su edad fisiológica, puesto a que la eficiencia fotosintética incrementa con relación a la edad de las hojas en exposición a la luz. Carranza (2014) sostiene que el contenido de clorofila está determinado por el estado de madurez de las hojas. De igual manera, Daymond y Hadley (2004) señalan que las condiciones ambientales controladas y la exposición de los genotipos a la baja tasa de luminosidad favorece la variación de la clorofila en el tiempo, y que la variación de la clorofila en condiciones de invernadero se debe a la interacción de la temperatura y la luz.

Cuadro 7. Contenido de clorofila de hoja en híbridos interclonales de Theobroma cacao L., en etapa de desarrollo inicial (3meses) y en sitio definitivo

\begin{tabular}{lcccc}
\hline \multirow{2}{*}{ Cruzamientos } & \multicolumn{4}{c}{ Clorofila (SPAD) } \\
\cline { 2 - 5 } & 30 días & 60 días & 90 días & 120 días \\
\hline L11-H19 x EET-103 & $34.9( \pm 3.8) \mathrm{c}$ & $36.3( \pm 3.1) \mathrm{b}$ & $37.3( \pm 2.6) \mathrm{b}$ & $42.7( \pm 0.9) \mathrm{b}$ \\
EET-558 x EET-103 & $36.8( \pm 2.7) \mathrm{a}$ & $37.6( \pm 2.2) \mathrm{a}$ & $38.4( \pm 1.7) \mathrm{a}$ & $43.7( \pm 0.2) \mathrm{b}$ \\
EET-558 x L11-H19 & $36.0( \pm 2.2) \mathrm{a}$ & $36.8( \pm 1.7) \mathrm{b}$ & $37.8( \pm 1.5) \mathrm{a}$ & $41.6( \pm 0.3) \mathrm{b}$ \\
EET-103 x L11-H19 & $36.5( \pm 2.7) \mathrm{a}$ & $37.3( \pm 1.9) \mathrm{a}$ & $38.0( \pm 1.6) \mathrm{a}$ & $42.3( \pm 0.4) \mathrm{b}$ \\
L11-H19 x CCN-51 & $34.5( \pm 2.9) \mathrm{c}$ & $35.9( \pm 2.0) \mathrm{b}$ & $37.1( \pm 1.6) \mathrm{b}$ & $43.5( \pm 0.6) \mathrm{b}$ \\
L11-H19 x EET-558 & $35.3( \pm 2.4) \mathrm{b}$ & $36.7( \pm 1.7) \mathrm{a}$ & $37.8( \pm 1.3) \mathrm{a}$ & $43.7( \pm 0.5) \mathrm{b}$ \\
EET-103 x CCN-51 & $34.5( \pm 3.6) \mathrm{c}$ & $35.8( \pm 2.8) \mathrm{b}$ & $36.9( \pm 2.3) \mathrm{b}$ & $42.8( \pm 0.6) \mathrm{b}$ \\
EET-558 x CCN-51 & $35.8( \pm 2.5) \mathrm{b}$ & $36.9( \pm 2.0) \mathrm{a}$ & $38.0( \pm 1.6) \mathrm{a}$ & $45.4( \pm 0.7) \mathrm{a}$ \\
EET-103 x EET-558 & $32.1( \pm 4.1) \mathrm{d}$ & $33.6( \pm 3.4) \mathrm{c}$ & $34.9( \pm 2.9) \mathrm{c}$ & $43.6( \pm 0.8) \mathrm{b}$ \\
CCN-51 x EET-103 & $32.1( \pm 3.2) \mathrm{d}$ & $35.8( \pm 2.8) \mathrm{b}$ & $36.7( \pm 2.5) \mathrm{b}$ & $45.5( \pm 0.9) \mathrm{a}$ \\
\hline$\overline{\mathrm{X}} \pm$ Sd & $34.8 \pm 1.6$ & $36.2 \pm 1.1$ & $37.2 \pm 1.0$ & $43.5 \pm 1.2$ \\
\hline $\mathrm{L}$ & &
\end{tabular}

Letras distintas indican diferencia estadística, Tukey $(\mathrm{p} \leq 0.05)$. 


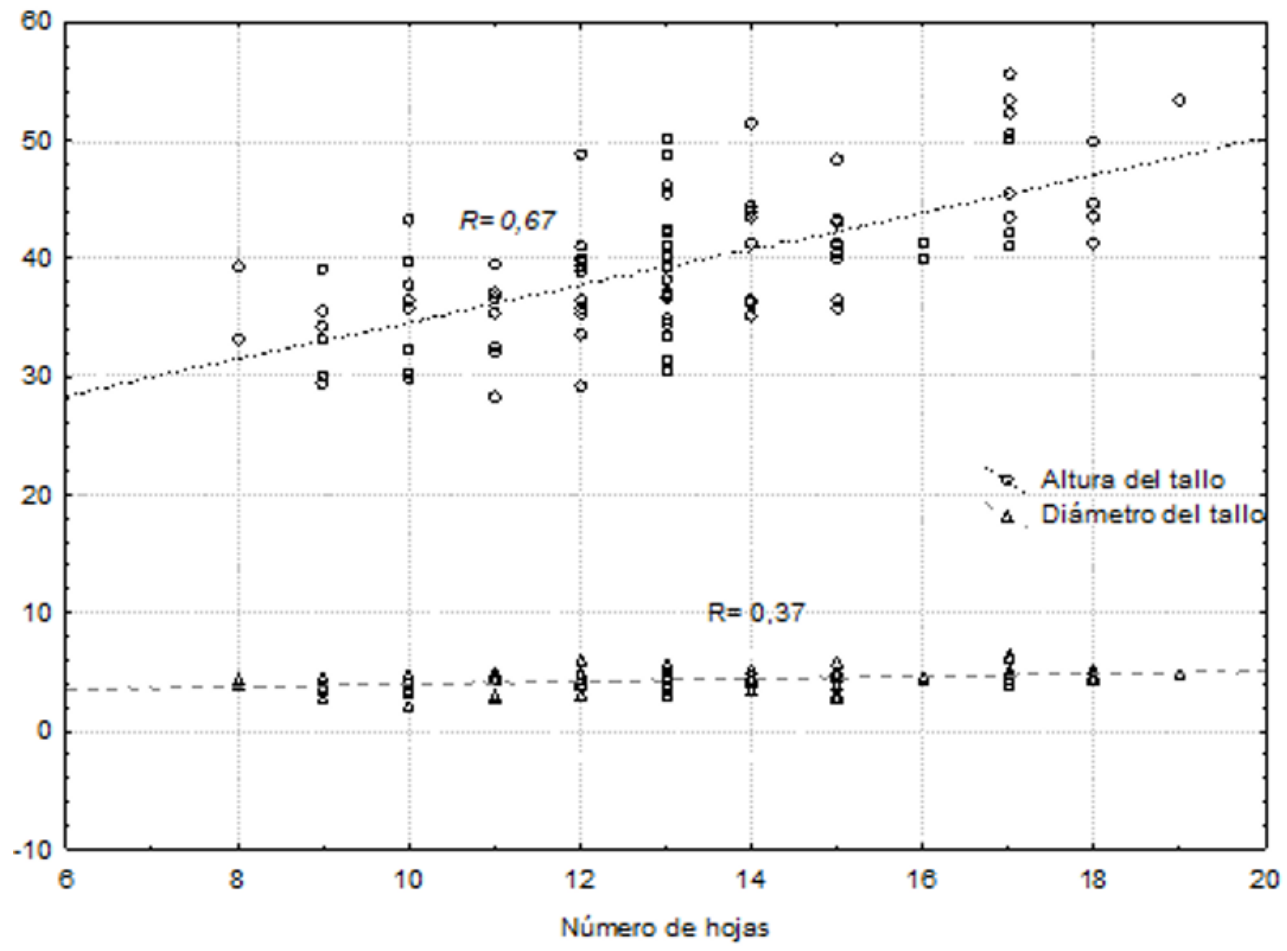

Figura 1. Correlación entre el número de hojas y las variables altura y diámetro del tallo en plántulas de Theobroma cacao a los 4 meses en vivero. Altura del tallo $=$ $19,0153+1,5586 * x, p=0,000 ;$ Diámetro del tallo $=2,7809+0,1153 * x, p=0,000$.

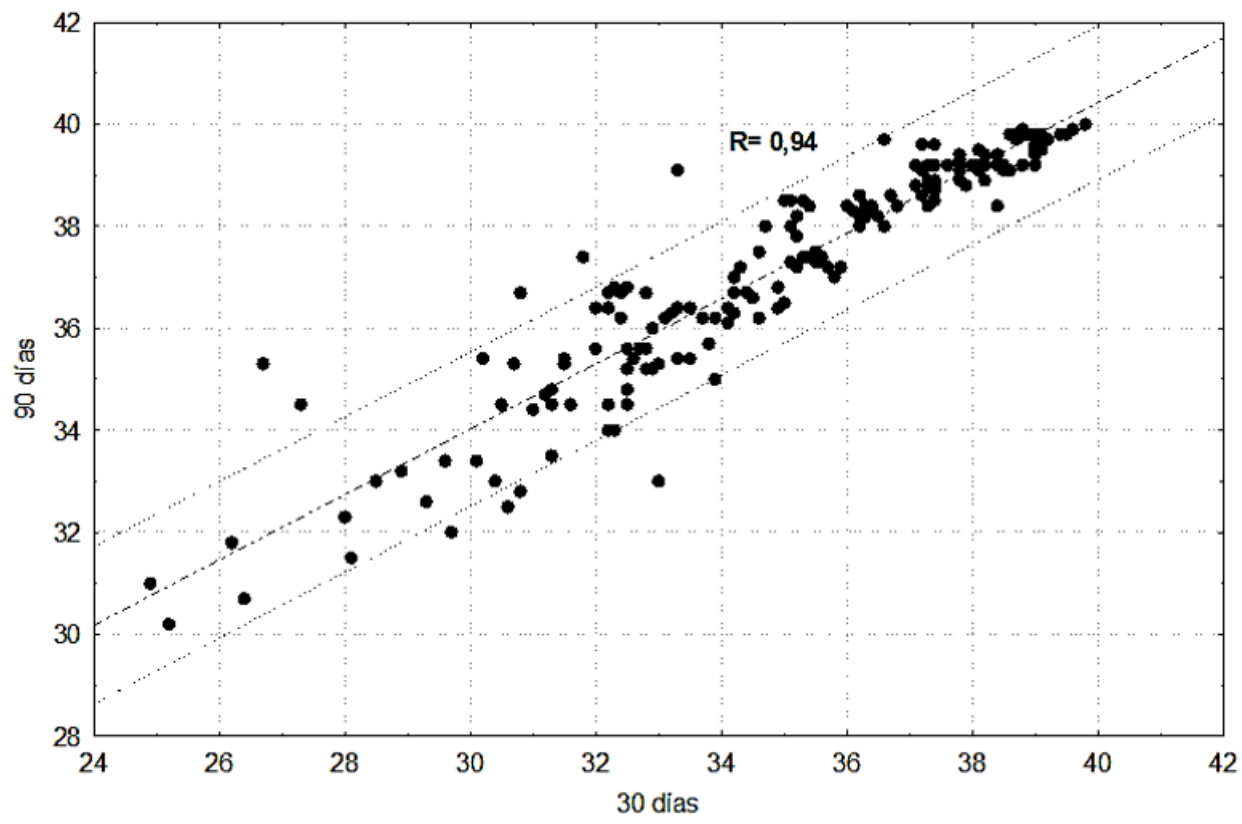

Figura 2. Correlación de clorofila en plántulas de Theobroma cacao $\mathrm{L}$ a los 3 meses en vivero. 90 días $=14,8133+0,6404 * x$; 0,95 Pred.Int. 


\section{Conclusiones}

$\mathrm{L}$ a germinabilidad en $T$. cacao es alta al someter a las semillas a un proceso de desmucilado y extracción del epispermo, pues esto facilita la apertura de los cotiledones y emergencia radicular. Se evidenció variabilidad en diámetro, altura, número de hojas y contenido de clorofila entre las progenies evaluadas. La mejor habilidad combinatoria general la presentó el clon EET-103 como madre y el EET-558 como padre, aunque se recomienda evaluar progenies en el campo y determinar posibles indicadores para realizar selección en vivero. Según nuestros resultados, el contenido de clorofila en las hojas está fuertemente correlacionado con la edad fisiológica de las plántulas, aunque también hay variación intra e interfamiliar por lo que se recomienda su estudio como un posible indicador de superioridad en genotipos promisorios.

\section{Literatura citada}

Acebo, M. (2016). Industria de Cacao. Escuela Superior Politécnica del Litoral (ESPAE), 1, 1-42.

Adu, M., Asare, P., Yawson, D., \& Taah, K. (2017). Demucilaging Freshly Stored Seeds of Cocoa (Theobroma cacao L.) Improves Seedling Emergence and Growth. Journal of Botany, 1-12. https://doi. org $/ 10.1155 / 2017 / 1938359$

Almeida J. (2012). Respuestas ecofisiológicas de cacao (Theobroma cacao L..) a diferentes intensidades lumínicas. Tesis para optar por el título de Licenciado en Biología, Universidad Central de Venezuela. 69 p. http:// saber.ucv.ve/jspui/handle/123456789/9139

Camacho, M., Cabrera, F., \& Garcia, D. (2006). Efectos Hetéroticos y habilidad combinatoria para el rendimiendo por planta en Cucurbita moschata DUCH. Ex Poir. Fac. Nal.Agr.Medellin, 59 (1), 3105-3121.

Carranza, M. (2014). Utilización de la espectro radiometría para el estudio del estado nutricional y fisiológico en plántulas de Jatropha curcas L., a nivel de vivero. Instituto Tecnológico de Costa Rica, Escuela de Ingeniería Forestal, 2014.

Carvalho, O., Pelacani, C., De Souza, M., \& Crepaldi, C. (2006). Crecimiento inicial de plantas de licuri (Syagrus coronata (mart) becc) en diferentes nieveis de luminosidade. Árvore, 30 (3), 351-357.

Contreras, F., \& Velázquez, B. (1999). Efecto de la intensidad de luz sobre el crecimiento en altura y producción de materia seca en plántulas de Pinus ayacahuite var. veitchii. Foresta Veracruzana, 1 (2), 25-30.

Cuéllar, N., \& Herrera, J. (2010). Evaluación de respuestas fisiológicas de la planta arbórea Hibiscus rosasinensis L (Cayeno) en condiciones de campo y vivero. Corpoica Ciencia y Tecnología Agropecuaria, 11, 61-72.
Daymond, A., \& Hadley, P. (2004). The effects of temperature and light integral on early vegetative growth and chlorophyll fluorescence of four contrasting genotypes of cacao (Theobroma cacao). Annals of Applied Biology, 145(3), 257-262. https://doi. org/10.1111/j.1744-7348.2004.tb00381.x

Ibarra, L., \& Reyes, R. (2015). Crecimiento en vivero de las palmas aceiteras Elaeis oleifera x Elaeis guineensis y Elaeis guineensis x Elaeis guineensis en Tumaco Colombia. Corpoica, 16 (2), 12.

Llerena, T., Bermeo, C., \& Plaza, P. (2017). Evaluación de diferentes tipos de sustratos en vivero de cacao (Theobroma cacao L). IJSEI, 03(01), 156-165.

López, S., \& Gil, A. (2017). Características germinativas de semillas de Theobroma cacao L (Malvaceae). Arnaldoa, 24(2), 609-618. https://doi.org//doi.org/10.22497/ arnaldoa.242.24212 ISSN:

Mena, M., \& García, C. (2017). Eficiencia reproductiva y receptividad estigmática en clones de cacao ( Theobroma cacao L.) en la zona central del Litoral ecuadoriano. Ganador del segundo concurso MAGAP, 2017. https:// fliphtml5.com/ijia/fukf

Motomiya, A., Molin, J., \& Chiavegato, E. (2009). Utilização de sensor óptico ativo para detectar deficiência foliar de nitrogênio em algodoeiro. Revista Brasileira de Engenharia Agrícola e Ambiental, 13 (67), 137-145. https://doi.org/10.1590/S1415-43662009000200005

Ortega, L., Sánche, J., Díaz, R., \& Ocampo, J. (2010). Efectos de diferentes sustratos en el crecimiento de plantulas de tomate (Lycopersicum esculentum Mill). Ra Ximhai, 6 (3), 365-372.

Quiroz, J., \& Amores, F. (2002). Rehabilitación de cacaotales tradicionales. Guayaquil, Ecuador: INIAP, Estación Experimental Boliche, Programa Nacional de Cacao. (Boletín Técnico no. 150). 16 p.

Sánchez, D., Proaño, O. H., Quiñónez, J. V. T. L. C., Matute, A. M., \& Morán, V. V. (2016). Potencial productivo de clones experimentales de cacao tipo "Nacional “. $L a$ Técnica, 14, 24-29.

Sarmiento, S., Gamboa, J., \& Velásquez, J. (2011). Desempeño agronómico de tres clones de cacao en fase de vivero en la Amazonía colombiana. Ingieneria \& Amazonia, 4 (1), 39-47. 